\title{
Exercise Therapy on Fatigue, Sleep Quality, and Quality of Life in Children Undergoing Umbilical Cord Blood Transplantation
}

\author{
Lu Huang ${ }^{1}$, Yao-Hua $\mathrm{Wu}^{1}$, Yun $\mathrm{Wu}^{1}$, Ying-Ying Wang ${ }^{1}$, Cheng-Zhen Jia ${ }^{1}$, Xue-Mei Liu ${ }^{1}$, \\ and Dao-Qin Wang ${ }^{1}$ \\ ${ }^{1}$ University of Science and Technology of China
}

October 30, 2020

\begin{abstract}
Background: Children undergoing umbilical cord blood transplantation (UCBT) have many treat-related severe side effects, which affects their quality of life (QoL) seriously. Exercise can help mitigate fatigue, improve sleep quality and QoL, however, the effects on children undergoing UCBT is limited. Procedure: Forty-eight children patients submitted to UCBT were divided into two groups by convenience sampling. The exercise therapy group performed abdominal breathing training combined with active joint movement program 3 days a week during hospitalization. The control group received routine exercise education without any scheduled programs. The fatigue, sleep quality, and QoL were evaluated at the date of hospital admission and discharge, and the differences between two groups were estimated using Student t test. Results: The exercise therapy group showed higher scores of general fatigue, rest fatigue, cognitive fatigue and the Pediatric Quality of Life Inventory (PedsQL) Multidimensional Fatigue Scale (MFS) total score, but no significant differences were found between two groups ( $\left.\mathrm{p}_{\llcorner} 0.05\right)$. There was an improvement in the sleep quality, verified by a reduction in the global score, subjective sleep quality, sleep latency, sleep efficiency, and daytime dysfunction ( $\mathrm{p}_{\mathrm{i}} 0.05$ ), analysis of the PedsQL 3.0 Cancer Module also revealed an improvement in procedure anxiety, worry, cognition, appearance, communication and total score in the exercise therapy group ( $\left.\mathrm{p}_{i} 0.05\right)$. Conclusion: Our prospective study demonstrates that exercise therapy is safe and feasible for children undergoing UCBT, and it has beneficial effects on sleep quality and QoL.
\end{abstract}

\section{Exercise Therapy on Fatigue, Sleep Quality, and Quality of Life in Children Undergoing Um- bilical Cord Blood Transplantation}

Lu Huang, $\mathrm{MD}^{1,2}$, Yao-Hua $\mathrm{Wu}, \mathrm{PhD}^{1 *}$, Yun Wu, BA ${ }^{2}$, Ying-Ying Wang, BA ${ }^{2}$, Cheng-Zhen Jia, BA ${ }^{2}$, Xue-Mei Liu, BA ${ }^{2}$, Dao-Qin Wang, BA ${ }^{2}$

${ }^{1}$ School of management, University of Science and Technology of China, Hefei ,230026, China

${ }^{2}$ Hematology Department, the First Affiliated Hospital of University of Science and Technology of China, Hefei ,230001, China

${ }^{*}$ Correspondence to: Yao-Hua Wu, email: wuyh@ustc.edu.cn

\section{Abstract}

Background: Children undergoing umbilical cord blood transplantation (UCBT) have many treat-related severe side effects, which affects their quality of life (QoL) seriously. Exercise can help mitigate fatigue, improve sleep quality and QoL, however, the effects on children undergoing UCBT is limited.

Procedure: Forty-eight children patients submitted to UCBT were divided into two groups by convenience sampling. The exercise therapy group performed abdominal breathing training combined with active joint 
movement program 3 days a week during hospitalization. The control group received routine exercise education without any scheduled programs. The fatigue, sleep quality, and QoL were evaluated at the date of hospital admission and discharge, and the differences between two groups were estimated using Student $\mathrm{t}$ test.

Results: The exercise therapy group showed higher scores of general fatigue, rest fatigue, cognitive fatigue and the Pediatric Quality of Life Inventory (PedsQL) Multidimensional Fatigue Scale (MFS) total score, but no significant differences were found between two groups $(p$ i0.05). There was an improvement in the sleep quality, verified by a reduction in the global score, subjective sleep quality, sleep latency, sleep efficiency, and daytime dysfunction $(p ; 0.05)$, analysis of the PedsQL 3.0 Cancer Module also revealed an improvement in procedure anxiety, worry, cognition, appearance, communication and total score in the exercise therapy group $(p \nmid 0.05)$. Conclusion: Our prospective study demonstrates that exercise therapy is safe and feasible for children undergoing UCBT, and it has beneficial effects on sleep quality and QoL.

\section{KEYWORDS}

Exercise Therapy; Undergoing Umbilical Cord Blood Transplantation; Fatigue; Sleep Quality; Quality of Life

\section{INTRODUCTION}

Umbilical cord blood transplantation (UCBT) has been a valuable alternative stem cell sources for treating malignant and nonmalignant hematopoietic disorders, especially for patients who lack a matched family donor. ${ }^{1}$ With improving survival rates, there has been an increase in the number of pediatric patients performed UCBT. However, due to the disease itself and the side effects of UCBT treatment, children often suffer from slower immune recovery, high infection and bleeding rates,and a lower incidence of graftversus-host disease (GVHD), ${ }^{1,2}$ in addition, the development of pain, fatigue, anxiety, depression, and sleep disruption are symptoms that directly affect the physical fitness and overall quality of life (QoL). ${ }^{3}$

Exercise interventions not only help to promote physical performance, but also be an effective strategy to improve psychological well-being in patients with hematologic malignancies. ${ }^{4-7}$ In adult hematopoietic stem cell transplantation (HSCT) recipients, physical exercise may positively influence their muscle strength, fatigue, and global QoL. ${ }^{8-9}$ In addition, two systematic review revealed that exercise can improve cancerrelated sleep disturbance, and sleep outcomes in cancer patients with poor sleep quality. ${ }^{10-11}$

The positive effects of exercise conducted on adult HSCT were well known, However, the effects of exercise interventions for childhood cancer participants are not yet convincing. ${ }^{12}$ In addition, a meta-analysis performed on children and adolescents patients submitted to HSCT showed that the heterogeneity between studies was high, the sample size was small and the methodological rigor was low. ${ }^{4}$ Therefore, the influences of exercise in children with cancer, the optimal physical exercise program for children undergoing HSCT is still needed to clarify.

During the treatment of UCBT, especially after conditioning regimen, the platelet count of patients is always below $20,000 / \mu \mathrm{L}$, to avoid exercise-induced bleeding, the recommended physical exercises for patients with thrombocytopenia is low intensity activities without resistance. ${ }^{13}$ Hence, the present prospective study was the first time to compare an abdominal breathing training combined with active joint movement program with routine exercise education in children UCBT recipients. The objective of our study was to investigate the feasibility of exercise therapy in the setting of UCBT and the effects on fatigue, sleep quality, and QoL.

\section{METHOD}

\subsection{Study design and setting}

The study was a prospective, not-randomized study to assess changes in fatigue, sleep quality, and QoL in children patients undergoing UCBT. In all, 48 patients receiving UCBT were screened by convenience sampling. Enrollment occurred within 3 days after admission, and the demographic data were captured at the same time. All questionnaires were completed at the date of hospital admission (T1) and discharge(T2) 
by the patients independently and without supervision. The study was approved by the ethical commission of the first affiliated hospital of university of science and technology of China. All participants gave written informed consent.

\subsection{Subjects and intervention}

Children with malignant hematopoietic diseases were considered eligible for the study. Inclusion criteria were 8 to 17 years of age, able to read and speak Chinese, with normal cognitive function, and the ability to provide informed consent. Exclusion criteria were uncontrolled cardiovascular, orthopedic, or neurological deficits that can't afford the performance tests. Exit criteria were failure of cord blood stem cell implantation or presence of serious complications (such as uncontrolled infection, III-IV hemorrhagic cystitis or acute GVHD).

Participants in the control group receiving routine exercise education without any scheduled exercise programs performed during hospitalization. Participants in the exercise therapy group started exercising from the admission to discharge in individual sessions and under the supervision of a physiotherapist. Exercise therapy was performed for 5-6 weeks, on 3 days a week. The mean training time was 15-20 minutes a day, included abdominal breathing training, neck movements, shoulder flexion, abduction, adduction, and extension, elbow flexion and extension, wrist flexion and extension, knuckle flexion and extension, hip flexion and extension, straight-leg raising, knee flexion and extension, ankle dorsiflexion and plantar flexion, and trunk bridge-style movement. If the participants showed signs of infection (body temperature ¿ $38^{\circ} \mathrm{C}$ ), anemia (hemoglobin $<6 \mathrm{~g} / \mathrm{dL}$ ), thrombocytopenia (platelet count $<10,000 / \mu \mathrm{L}$ ), or severe pain, nausea and vomiting, and dizziness, the exercise therapy would postponed, when their symptoms relieved, hemoglobin and platelet counts reached the standard after transfusion of blood products, the missed exercise therapy made up.

\subsection{Assessment}

\section{Fatigue}

Participants completed the child self-reports of the validated Chinese version of the Pediatric Quality of Life Inventory (PedsQL) Multidimensional Fatigue Scale (MFS). ${ }^{14}$ This 18 -item self-report questionnaire including three domains, general fatigue, rest fatigue, and cognitive fatigue. The items were rated using a 5-point Likert scale ranging from 0 to 4 , reversed and rescaled to 0-100 scale, with lower scores indicate more symptoms of fatigue. ${ }^{15}$ The Cronbach's alpha of Chinese version of PedsQL MFS varied from 0.92 to 0.93 for the child report. ${ }^{14}$

\section{Sleep quality}

Participants completed the Chinese version of the Pittsburgh Sleep Quality Index (PSQI). ${ }^{16}$ The PSQI including seven domains, and then these are combined to assess the global sleep quality. The total global PSQI score is ranging from 0 to 21, the lower global score the better sleep quality, and the global score greater than 5 indicates poor sleep quality. ${ }^{17}$ The Cronbach's alpha of the Chinese version of PSQI varied from 0.82 to $0.83 .{ }^{16}$

\section{Quality of life}

Participants completed the child self-reports of the validated Chinese version of the PedsQL 3.0 Cancer Module. ${ }^{18}$ The PedsQL 3.0 Cancer Module is a 27 -item measure consists of eight subscales including pain, nausea and vomiting, and so on. The items were rated using a 5-point Likert scale ranging from 0 to 4 , reversed and transformed into 0 to 100 scale, a mean score is obtained by averaging the 27 items, with higher scores indicate better QoL. ${ }^{15}$ The Cronbach's alpha of Chinese version of PedsQL 3.0 Cancer Module varied from 0.84 to 0.99 for the child report. ${ }^{18}$

\subsection{Data analysis}

Statistical Package for SPSS version 16.0 (SPSS, Chicago, IL, USA) was carried out to analyze data, and all the data were checked for normal distribution and homogeneity of variance before analysis. For baseline 
comparisons, the metric data were explored with Student t test, the ordinal data were compared by MannWhitney test, and categorical data were examined by chi-square test. For patient-rated outcomes, the differences and their significance at 2 fixed time points between two groups were estimated using Student $\mathrm{t}$ test. When $p \nmid 0.05$ the results of comparative were considered as statistically significant.

\section{RESULTS}

Of the 48 patients who met the inclusion criteria and agreed to participate, 4 patients (2 in each group) withdraw from the study because the presence of serious complications (III-IV acute GVHD). The patient characteristics of both two groups are shown in Table 1. 44 patients whose mean age were $10.76 \pm 2.53$ years in control group and $11.04 \pm 2.58$ years in exercise therapy group $(p i 0.05)$. The mean adherence to exercise therapy was $72.22 \%$, and there was no exercise-induced bleeding during hospitalization.

The results of the PedsQL MFS are presented in Table 2. The scores of PedsQL MFS scale were dropped in both two groups after UCBT, except for the cognitive fatigue in the exercise therapy group. There was no significant differences between two groups at T2, however, the scores of general fatigue, rest fatigue, cognitive fatigue, and PedsQL MFS total scale were higher in the exercise therapy group ( $p$ i 0.05$)$.

The results of PSQI are presented in Table 3. All the children had poorer sleep quality after UCBT in both groups, with the global sleep quality score 5 . However, the exercise therapy group had an improvement in the sleep quality, verified by a reduction in the global score, subjective sleep quality, sleep latency, sleep efficiency, and daytime dysfunction $(p ; 0.05)$.

The results of the PedsQL 3.0 Cancer Module are presented in Table 4. The exercise therapy group reached better results for procedure anxiety, worry, cognition, appearance, communication and total score than the

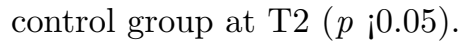

\section{DISCUSSION}

HSCT is associated with numerous transplant-related side effects, which may have a profound impact on a patient's physical functioning and overall QoL during hospitalization, especially for UCBT. However, most previous studies have reported that physical exercise training can mitigated the loss of condition after HSCT. ${ }^{4-9}$ Currently, the Clinical Oncology Society of Australia has recommended that exercise should be integrated into cancer care as part of standard practice. ${ }^{19}$ In this first prospective study conducted in the setting of UCBT, our results indicate that exercise therapy can improve sleep quality and QoL of the children who undergo UCBT.

In the present study, the fatigue of both groups was intensified after UCBT, except for the cognitive fatigue in exercise therapy group. The scores of PedsQL MFS scale were higher in the exercise therapy group at T2, however, there was no significant differences between two groups. Our observations were in agreement with other reports. ${ }^{20-22}$ The supervised high intensity exercise program was not effective at improving physical fitness and fatigue when compared with the controls. ${ }^{20}$ Physical activity had positive effects on the severity of fatigue in patients with cancer and HSCT recipients, ${ }^{21}$ however, no effects for exercise on fatigue was found in after HSCT subgroup was reported. ${ }^{22}$

HSCT recipients often accompanied with inadequate and irregular sleep during hospitalization, ${ }^{23}$ and diminished sleep quality can exacerbate other QoL concerns. ${ }^{24}$ In our study, all patients had poorer sleep quality after UCBT in both groups, with the global sleep quality score; 5 . Especially the mean sleep quality scores of the control group at T2 were poorer than other types of allogeneic HSCT, ${ }^{24}$ at which point UCBT recipients were experiencing more treatment-related toxicities and complications. However, Exercise positively impacts sleep quality and quantity, ${ }^{25}$ participants in the exercise therapy reported less sleep disturbance, verified by a reduction in the global sleep quality score, subjective sleep quality, sleep latency, sleep efficiency, and daytime dysfunction in our results.

As assessing QoL level of children, higher scores of PedsQL 3.0 Cancer Module were achieved using the exercise therapy when compared to routine exercise education. Exercise during pediatric HSCT contributes 
to prevention of treatment-related loss of physical function, ${ }^{26}$ and exercise program under supervision has beneficial effects on children's QoL level. ${ }^{27}$ We suggest that exercise therapy can be effective in clinical practice, since patients undergoing UCBT are accompanied by variety of symptoms. In addition, we believe that exercise therapy for children may help to reduce the feeling of social isolation and loneliness. Therefore, it is recommended to communicate with children and accompany them more.

The strengths of our study are the prospective trial, a controlled study, and the inclusion of only UCBT recipients. The limitations of this study comprise a small sample size, the use of a convenience sample, not a randomized study, and without a follow-up after discharge. Thus, there might have biased in our results, a larger multicenter randomized study with a longer follow-up is needed to confirm. However, to the best of our knowledge, this is the first study to explore the feasibility of exercise therapy for children undergoing UCBT.

In conclusion, this prospective study demonstrates that an abdominal breathing training combined with active joint movement program is well tolerated. An inpatient, supervised, and personalized exercise program can be effective in the setting of UCBT. We are convinced that exercise therapy has beneficial effects on sleep quality and QoL for children submitted to UCBT.

\section{CONFLICT OF INTEREST}

The authors declare that they have no conflict of interest.

\section{ACKNOWLEDGEMENTS}

We are grateful to our study participants and appreciate our physical therapists for helping us conduct the study.

\section{REFERENCES}

1.Yun HD, Varma A, Hussain MJ, et al. Clinical Relevance of Immunobiology in Umbilical Cord Blood Transplantation. J Clin Med.2019;8:1968.

2.Gabelli M, Veys P, Chiesa R. Current status of umbilical cord blood transplantation in children. Br J Haematol. 2020;190:650-683.

3.Guilcher GM. Hematopoietic Stem Cell Transplantation in Children and Adolescents. Pediatr Rev. 2016;37:135-144.

4. Martha BA, Vacchi CO, Fattori RA, et al. Effect of physical exercise on the functional capacity of children and adolescents submitted to transplantation of hematopoietic stem cells-A systematic review with meta-analysis. J Child Health Care. 2020;1367493520903626.

5. Wiskemann J, Huber G. Physical exercise as adjuvant therapy for Patients undergoing hematopoietic stem cell transplantation. Bone Marrow Transplant. 2008; 41:321-329.

6. Bogg TFT, Broderick C, Shaw P, et al. Feasibility of an inpatient exercise intervention for children undergoing hematopoietic stem cell transplant. Pediatr Transplant.2015;19:925-931.

7. Schumacher H, Stuwe S, Kropp P, et al. A prospective, randomized evaluation of the feasibility of exergaming on patients undergoing hematopoietic stem cell transplantation. Bone Marrow Transplant. 2018;53:584-590.

8. Persoon S, Kersten MJ, van der Weiden K, et al. Effects of exercise in patients treated with stem cell transplantation for a hematologic malignancy: a systematic review and meta-analysis. Cancer Treat Rev. 2013; 39: 682-690.

9. Liang Y, Zhou M, Wang F, et al. Exercise for physical fitness, fatigue and quality of life of patients undergoing hematopoietic stem cell transplantation: a meta-analysis of randomized controlled trials. Jpn J Clin Oncol. 2018;48:1046-1057. 
10. Takemura N, Cheung DST, Smith R, et al. Effectiveness of aerobic exercise and mind-body exercise in cancer patients with poor sleep quality: A systematic review and meta-analysis of randomized controlled trials. Sleep Med Rev. 2020;53:101334.

11. Yao LQ, Tan JY, Turner C, et al. Traditional Chinese exercise for cancer- related sleep disturbance: A systematic review and descriptive analysis of randomized controlled trials. Complement Ther Clin Pract. 2020;40:101197.

12. Braam KI, van der Torre P, Takken T, et al. Physical exercise training interventions for children and young adults during and after treatment for childhood cancer. Cochrane Database Syst Rev. 2016;3:CD008796.

13.Morishita S, Nakano J, Fu JB, et al. Physical exercise is safe and feasible in thrombocytopenic patients with hematologic malignancies: a narrative review. Hematology.2020;25; 95-100.

14. Varni JW, Burwinkle TM, Katz ER, et al. The PedsQL in pediatric cancer:reliability and validity of the Pediatric Quality of Life Inventory Generic Core Scales, Multidimensional Fatigue Scale, and Cancer Module. Cancer. 2002;94:2090-2106.

15. XQ Bu, QM Ye, K Liu, et al. The applicafion of Chinese version of PedsQL ${ }^{\mathrm{TM}}$ Multidimensional Fatigue Scale in children with leukemia. ZHONG GUO SHI YONG HU LI ZA ZHI. 2015;31:323-326.

16. Tsai PS, Wang SY, Wang MY, et al. Psychometric evaluation of the Chinese version of the Pittsburgh Sleep Quality Index (CPSQI) in primary insomnia and control subjects. Qual Life Res. 2005;14:1943-1952.

17.Buysse DJ, Reynolds CF, Monk TH, et al. The Pittsburgh Sleep Quality Index: A new instrument for psychiatric research and practice. Psychiatry Res. 1989;28:193-213.

18. Ji Y, Chen S, Li K, et al. Measuring health-related quality of life in children with cancer living in Mainland China: feasibility, reliability and validity of the Chinese Mandarin version of PedsQL 4.0 Generic Core Scales and 3.0 Cancer Module. Health Qual Life Outcomes.2011;9:103.

19. COSA. COSA Position Statement on Exercise in Cancer. 2018.

20. Persoon S, ChinAPaw MJM, Buffart LM, et al. Randomized controlled trial on the effects of a supervised high intensity exercise program in patients with a hematologic malignancy treated with autologous stem cell transplantation: results from the EXIST study. PLoS One. 2017;12: e0181313.

21. Oberoi S, Robinson PD, Cataudella D, et al. Physical activity reduces fatigue in patients with cancer and hematopoietic stem cell transplant recipients: A systematic review and meta-analysis of randomized trials. Crit Rev Oncol Hematol. 2018;122: 52-59.

22.Liang Y, Zhou M, Wang F, et al. Exercise for physical fitness, fatigue and quality of life of patients undergoing hematopoietic stem celltransplantation: a meta-analysis of randomized controlled trials. Jpn J Clin Oncol. 2018;48:1046-1057.

23. Hacker ED, Kapella MC, Park C, et al. Sleep Patterns During Hospitalization Following Hematopoietic Stem Cell Transplantation. Oncol Nurs Forum. 2015;42:371-379.

24. Nelson AM, Coe CL, Juckett MB, et al. Sleep quality following hematopoietic stem cell transplantation: longitudinal trajectories and biobehavioral correlates. Bone Marrow Transplant. 2014;49:1405-1411.

25.Medysky ME, Temesi J, Culos-Reed SN, et al. Exercise, sleep and cancer-related fatigue: Are they related? Neurophysiol Clin. 2017;47:111-122.

26. Wallek S, Senn-Malashonak A, Vogt L, et al. Impact of the initial fitness level on the effects of a structured exercise therapy during pediatric stem cell transplantation. Pediatr Blood Cancer. 2018;65. 
27. Yildiz Kabak V, Cetinkaya DU, Kuskonmaz B, et al. Effects of multimodal exercise on clinical status and patient-reported outcomes in children undergoing hematopoietic stem cell transplantation. Pediatr Hematol Oncol. 2019; 36:410-421.

\section{Hosted file}

Tables.pdf available at https://authorea.com/users/371506/articles/489835-exercise-therapyon-fatigue-sleep-quality-and-quality-of-life-in-children-undergoing-umbilical-cordblood-transplantation 